\title{
FAMILIAL COMBINED HYPERLIPIDEMIA: CURRENT KNOWLEDGE, PERSPECTIVES, AND CONTROVERSIES
}

\author{
Omar Yaxmehen Bello-Chavolla ${ }^{1,2}$, Anuar Kuri-García ${ }^{1}$, Monserratte Ríos-Ríos ${ }^{1}$, \\ Arsenio Vargas-VázQuez $Z^{1,2}$, Jorge Eduardo Cortés-Arroyo ${ }^{1,2}$, Gabriela TAPIA-GonzÁlez $^{1}$, \\ Ivette Cruz-Bautista ${ }^{1,3,4}$ and Carlos Alberto Aguilar-Salinas ${ }^{1,3,4 *}$
}

${ }^{1}$ Metabolic Diseases Research Unit and ${ }^{3}$ Department of Endocrinology and Metabolism, Instituto Nacional de Ciencias Médicas y Nutrición Salvador Zubirán, Mexico City, Mexico; 2MD/PhD (PECEM) Program, Facultad de Medicina, Universidad Nacional Autónoma de México; ${ }^{4}$ Tec Salud, Instituto Tecnológico y de Estudios Superiores de Monterrey, Monterrey, N.L., México

\begin{abstract}
Familial combined hyperlipidemia ( $F C H L)$ is the most prevalent primary dyslipidemia; however, it frequently remains undiagnosed and its precise definition is a subject of controversy. FCHL is characterized by fluctuations in serum lipid concentrations and may present as mixed hyperlipidemia, isolated hypercholesterolemia, hypertriglyceridemia, or as a normal serum lipid profile in combination with abnormally elevated levels of apolipoprotein B. FCHL is an oligogenic primary lipid disorder, which can occur due to the interaction of several contributing variants and mutations along with environmental triggers. Controversies surrounding the relevance of identifying $\mathrm{FCHL}$ as a cause of isolated hypertriglyceridemia and a differential diagnosis of familial hypertriglyceridemia are offset by the description of associations with USF1 and other genetic traits that are unique for FCHL and that are shared with other conditions with similar pathophysiological mechanisms. Patients with FCHL are at an increased risk of cardiovascular disease and mortality and have a high frequency of comorbidity with other metabolic conditions such as type 2 diabetes, non-alcoholic fatty liver disease, steatohepatitis, and the metabolic syndrome. Management usually requires lipidlowering therapy directed toward reducing cholesterol and triglyceride concentrations along with cardiovascular risk protection. In recent years, the number of research studies on FCHL has been decreasing, mainly due to a lack of recognition of its impact on disease burden and comorbidity and the complexity in identifying probands for studies. This creates areas of opportunity to develop research for FCHL in epidemiology, genetics, pathophysiology, therapeutics, and cardiovascular risk management, which are discussed in depth in this review. (REV INVEST CLIN. 2018;70:224-36)
\end{abstract}

Key words: Familial combined hyperlipidemia. Genetics. Apolipoprotein B.

Corresponding author:

*Carlos Alberto Aguilar-Salinas

Departamento de Endocrinología y Metabolismo

Instituto Nacional de Ciencias Médicas

y Nutrición Salvador Zubirán

Vasco de Quiroga, 15

Col. Belisario Domínguez Sección XVI, Del. Tlalpan $\quad$ Received for publication: 02-05-2018

C.P. 14080, Mexico City, Mexico

E-mail: caguilarsalinas@yahoo.com

Approved for publication: 13-06-2018 doi: $10.24875 /$ RIC.18002575 


\section{INTRODUCTION}

Familial combined hyperlipidemia ( $F C H L)$ is the most prevalent primary dyslipidemia, occurring in up to $1-3 \%$ of the general population and in $20-38 \%$ of patients with previous history of myocardial infarction $(\mathrm{MI})^{1}$. This disorder was simultaneously described by Goldstein et al. ${ }^{2}$, Hazzard et al., and Kwiterovich et al., who independently described it in different cohorts ${ }^{3}$. $\mathrm{FCHL}$ is characterized by fluctuations in serum lipid profile and a rather heterogeneous clinical presentation which can be alternatingly identified with mixed hyperlipidemia, isolated hypercholesterolemia, or hypertriglyceridemia in combination with abnormally high levels of apolipoprotein B (apoB) ${ }^{2,3}$. Certain ethnic groups are particularly susceptible to $\mathrm{FCHL}$, as demonstrated by Paramsothy et al. in a multiethnic cohort of 6814 participants in the United States, reporting a prevalence of $4.8 \%$ within Hispanics ${ }^{4,5}$. FCHL coexists with other metabolic diseases such as obesity, insulin resistance (IR), type 2 diabetes mellitus (T2D), hypertension, non-alcoholic fatty liver disease (NAFLD), and metabolic syndrome (MS) ${ }^{6}$. FCHL cases with metabolic comorbidities have remarkably higher apoB plasma levels compared to cases with a similar severity of IR. In addition, subjects with FCHL have a greater susceptibility to developing T2D and are thus at a higher cardiovascular risk in comparison to matched controls ${ }^{3,6}$.

Notably, the number of research studies focused on understanding the epidemiology, genetics, pathophysiology, and treatment of $\mathrm{FCHL}$ has been decreasing over the years as demonstrated by the number of related articles cited in PubMed since 2007 (Fig. 1).

Figure 1. Publication rate related to studies focused on familial combined hyperlipidemia in the scientific database PubMed until December 2017.

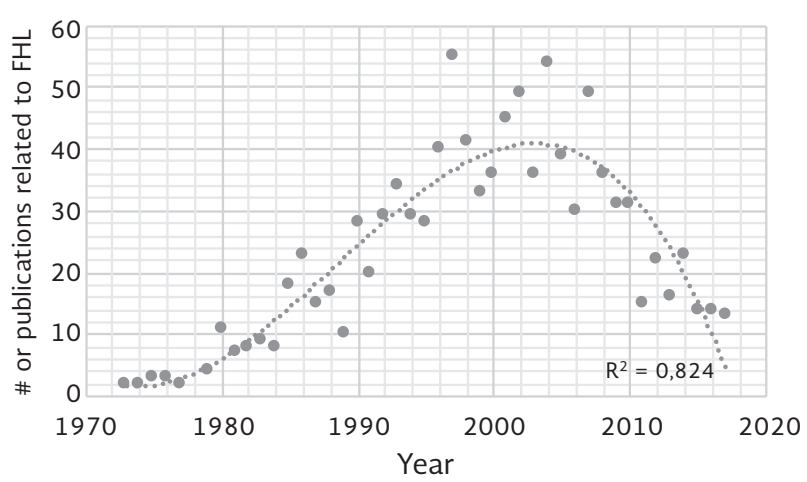

This could be attributable to the complex nature of the disease, heterogeneous clinical definitions, and inconsistent consensus in its defining traits, which makes comparisons across reports largely unfeasible, complicating precise estimates of FCHL epidemiology and its metabolic burden. In this review, we will focus on the most recent advances in understanding FCHL. We will also evaluate gaps in available knowledge and areas that lack sufficient information and call for further studies to describe fully comorbidity and cardiovascular risk associated to $\mathrm{FCHL}$.

\section{EVOLUTION OF FCHL DIAGNOSTIC CRITERIA}

Different diagnostic criteria have been proposed for FCHL over the years ${ }^{7}$. Classically, the phenotype to establish the diagnosis of FCHL comprised either isolated hypercholesterolemia or hypertriglyceridemia or a mixed lipid profile along with the first-degree family history of premature coronary artery disease (CAD), excluding other causes of dyslipidemia ${ }^{3}$. More recent criteria have also included elevated apoB levels as highly suggestive of FCHL (Table 1$)^{8}$.

Due to the oligogenic nature of the disease, genetic testing is not yet a possibility ${ }^{3}$, but diagnosis can be made based on a fluctuating lipid profile, increased apoB levels, and first-degree family history of mixed lipid disorders and premature cardiovascular disease (Fig. 2) ${ }^{9-11}$. Some limitations on these criteria include the low practicality of apoB measurements in everyday clinical settings in addition to interethnic differences in establishing the 90th percentile in both lipid and apoB measurements, which require populationspecific percentiles that might not always be available.

\section{GENETIC CHARACTERIZATION OF FCHL}

Initial genetic characterizations of $\mathrm{FCHL}$ defined it as a primary lipid disorder with autosomal dominant inheritance ${ }^{2}$; however, recent data suggest that $\mathrm{FCHL}$ is an oligogenic entity with variable penetrance ${ }^{11,12}$. Establishing a unified causative genetic trait in FCHL is complex partly due to its clinical variability and the difficulties in comparing FCHL studies with inconsistent diagnostic criteria. Recent findings describe multiple genetic alterations contributing to the observed 
Table 1. Changes in diagnostic criteria for FCHL throughout the years.

\begin{tabular}{|c|c|c|c|c|c|c|}
\hline Year & Study/Author & TG $(\mathrm{mmol} / \mathrm{l})$ & & CT $(\mathrm{mmol} / \mathrm{l})$ & ApoB (g/I) & Family history \\
\hline 1973 & Goldstein & $>95^{\text {th }}$ percentile & And & $>95^{\text {th }}$ percentile & - & $C A D<60$ years \\
\hline 1983 & Brunzell & $6.42 \pm 1.19$ & And & $2.53 \pm 1.17$ & $1.44 \pm 0.36$ & $C A D<60$ years \\
\hline 1999 & $\begin{array}{l}\text { EuroFam/ } \\
\text { Pajukanta }\end{array}$ & $>90^{\text {th }}$ percentile & Or & $>90^{\text {th }}$ percentile & - & Mixed hyperlipidemia \\
\hline 1999 & Dutch/Aouizerat & $>6.5$ & And & $>2.3$ & $>1.2$ & $\begin{array}{l}\text { Differing hyperlipidemia } \\
\text { in relative, CAD age } \\
<60 \text { years }\end{array}$ \\
\hline 2001 & $\begin{array}{l}\text { Consensus/ } \\
\text { Sniderman }\end{array}$ & - & - & $>1.5$ & $>75$ th percentile & $\begin{array}{l}\text { Hyperlipidemia in } \\
1^{\text {st }} \text { degree relative }\end{array}$ \\
\hline 2003 & $\begin{array}{l}\text { British mapping/ } \\
\text { Naoumova }\end{array}$ & $>95^{\text {th }}$ percentile & And & $>90^{\text {th }}$ percentile & - & $\begin{array}{l}\text { Hyperlipidemia in } 1^{\text {st }} \\
\text { and } 2 \text { nd degree relatives }\end{array}$ \\
\hline 2004 & $\begin{array}{l}\text { Dutch clinical/ } \\
\text { Veerkamp }\end{array}$ & $>6.0$ & And & $>1.5$ & $>1.2$ & $\begin{array}{l}\text { Hyperlipidemia in } \\
\text { degree relative }\end{array}$ \\
\hline 2004 & $\begin{array}{l}\text { Huertas- } \\
\text { Vazquez }\end{array}$ & $>90^{\text {th }}$ percentile & Or & $>90^{\text {th }}$ percentile & $>90^{\text {th }}$ percentile & $\begin{array}{l}\text { CAD }(\mathrm{MI})<60 \text { years in } \\
\text { proband or } 1 \text { st degree } \\
\text { relative and } \\
\text { One } 1^{\text {st }} \text { degree relative TG } \\
\text { or } \mathrm{CT}>90^{\text {th }} \text { percentile }\end{array}$ \\
\hline 2004 & Aguilar-Salinas & $>150 \mathrm{mg} / \mathrm{dL}$ & Or & $>200 \mathrm{mg} / \mathrm{dL}$ & > 90th percentile & $\begin{array}{l}\text { CAD }(\mathrm{MI})<60 \text { years } \\
\text { At least three different } \\
\text { family members: } \\
\text { one with } \\
\text { hypercholesterolemia, } \\
\text { one with } \\
\text { hypertriglyceridemia, } \\
\text { and one with mixed } \\
\text { hyperlipidemia }\end{array}$ \\
\hline 2005 & $\begin{array}{l}\text { GEM study/ } \\
\text { Wyszynski }\end{array}$ & - & - & $>75^{\text {th }}$ percentile & - & $\begin{array}{l}\text { Index case and one } \\
\text { relative with relevant } \\
\text { profile }\end{array}$ \\
\hline 2014 & Mata & $>200 \mathrm{mg} / \mathrm{dL}$ & And/or & $\begin{array}{c}<240 \mathrm{mg} / \mathrm{dL} \\
(\mathrm{LDL}>160 \\
\mathrm{mg} / \mathrm{dL})\end{array}$ & - & $\begin{array}{l}\text { Two or more family } \\
\text { members with } \\
\text { hypercholesterolemia, } \\
\text { hypertriglyceridemia, } \\
\text { or mixed hyperlipidemia }\end{array}$ \\
\hline
\end{tabular}

FCHL: familial combined hyperlipidemia, TG: triglycerides, CT: computed tomography, ApoB: apolipoprotein B, CAD: coronary artery disease, MI: myocardial infarction, LDL: low-density lipoprotein

*Adapted and modified from Wierzbicki AS (31).

clinical phenotype $2,3,11$. The fluctuating lipid profile characteristic of $\mathrm{FCHL}$ can be attributable to the interaction of cumulative large- and small-effect genetic variants that alter low-density lipoprotein-cholesterol (LDL-C) and triglycerides (TG) concentrations and contributing environmental factors (Fig. 3 ). These genetic alterations usually have independent segregation on different chromosomes, which impacts the degree of expression in affected family members, thus leading to heterogeneous clinical presentations even within the same kindred ${ }^{11}$.
Consistent susceptibility loci have been reported among individuals with FCHL from different ethnic backgrounds and have been mapped to chromosomes 1q21-23, 11p14.1-q12.1, and 16q22-24.113. An association of $\mathrm{FCHL}$ with the region in chromosome 1q21-1q231,14 has been consistently reported. This region includes several genes which might contribute to $\mathrm{FCHL}$ phenotype, including the upstream transcription factor 1 gene (USF1) ${ }^{1}$. USF1 encodes a transcription factor that regulates nearly 40 genes implicated in lipid and lipoprotein metabolism, as well as immune 
Figure 2. Proposed updated diagnostic algorithm for FCHL. TC: total cholesterol; TG: triglycerides; apoB: apolipoprotein B-100; CAD: coronary artery disease, FCHL: familial combined hyperlipidemia.

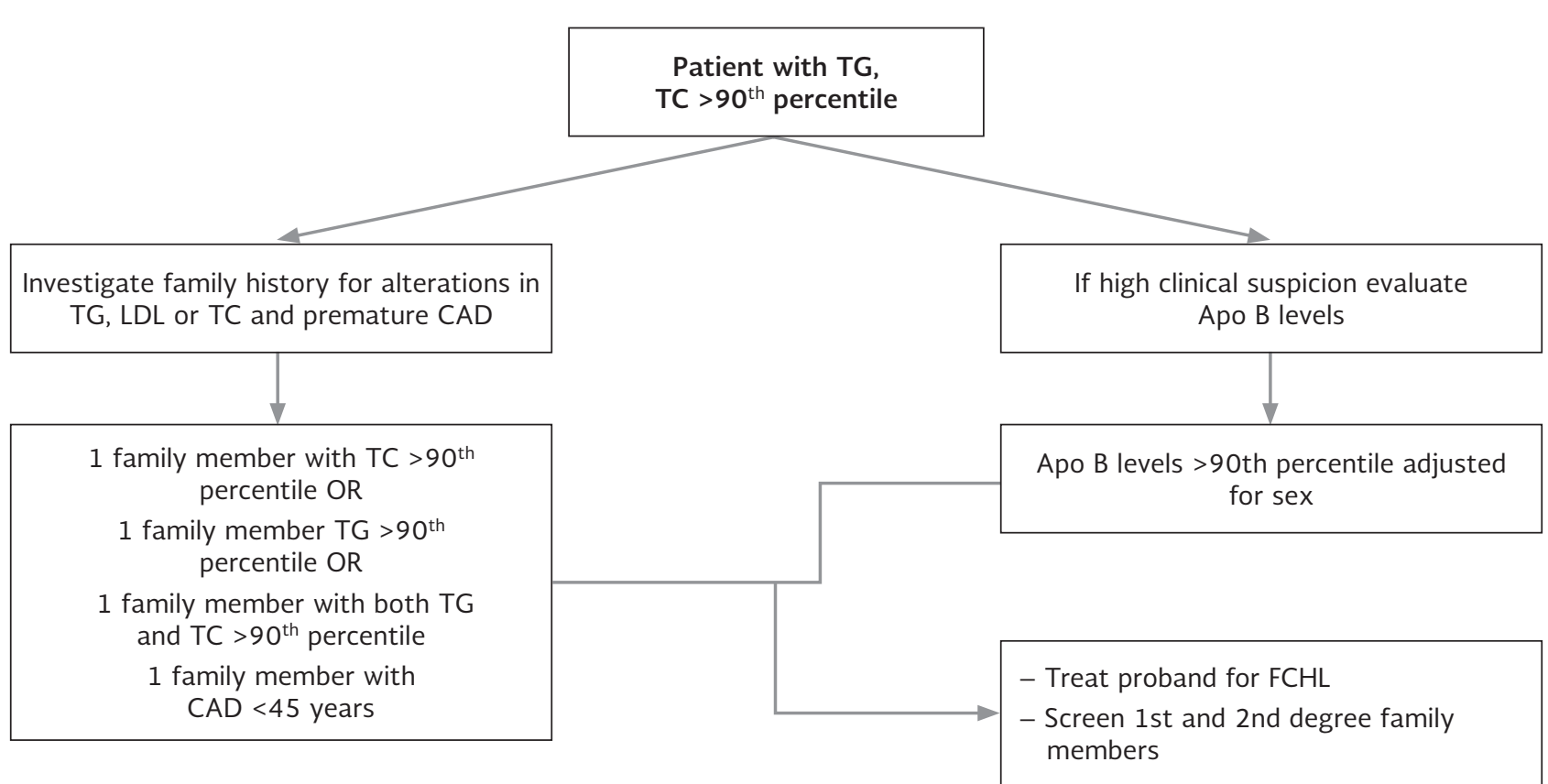

Figure 3. Genetics of familial combined hyperlipidemia (FCHL): The interplay of large-effect genetic mutations, cumulative smalleffect genetic variants, and environmental triggers contribute to developing the FCHL phenotype.

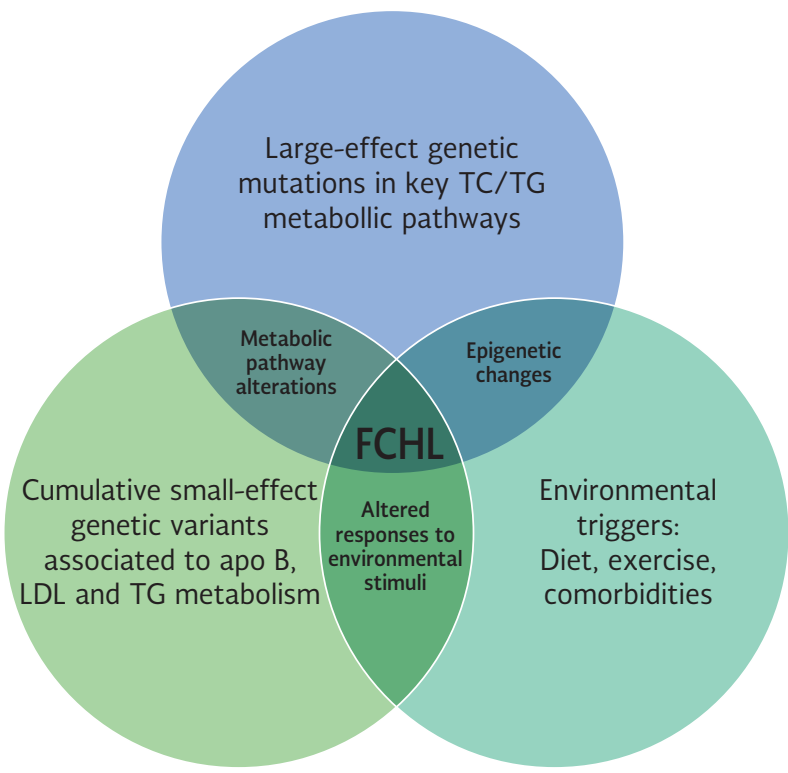

response, and is located $1.5 \mathrm{Mb}$ away from TXNIP, a gene linked to mixed hyperlipidemia in mice ${ }^{1,3}$. USF1 encodes for a basic helix-loop-helix leucine zipper transcription factor located in chromosome 1q23.3, which binds to a palindromic E-box sequence. USF1 was first described by Sawadogo et al. as a key component in adenovirus replication ${ }^{15}$ and its role as a regulator of lipid and glucose metabolism was later reported. USF1 has been shown to regulate expression of L-pyruvate kinase, fatty acid synthase, and glucokinase, as well as apoA-V, apoC-III, apoA-II, apoE, hormone-sensitive lipase, and other enzymes involved 
in lipid and carbohydrate metabolism ${ }^{16-19}$. Pajukanta et al. characterized USF1 as the major genetic trait of FCHL which was further demonstrated by HuertasVázquez et al. in Mexican population ${ }^{1,20}$.

Several single-nucleotide polymorphisms (SNP) have been associated with FCHL. A haplotype for USF1 associated with susceptibility for both FCHL and FHTG was identified in Finnish and Mexican families, with a stronger association for $\mathrm{FCHL}^{20}$. The SNP rs3737787 has been associated with differences in the expression of the target genes for USF1 in adipose tissue and lymphoblasts, as well as higher TG concentrations in Mexican and Finnish populations, and is the SNP most consistently associated to FCHL. A comparison of the expression of USF1 in muscle and adipose tissue identified 13 genes that are regulated by USF1, including FASD3, FABP2, FOLH1, MADD, NR1H3, CETP, LCAT, APOE, and PLTP21. Overall, GWAS have confirmed that subjects with $\mathrm{FCHL}$ have a high polygenic lipid score for associated LDL-C and TG variants and confirm the polygenic nature of the disease ${ }^{1,10}$.

Mutations in LDLR and PCSK9 have been associated to increased LDL-C levels in FCHL; however, identification of these mutations is not specific of $\mathrm{FCHL}$ and demonstrates the difficulty of distinguishing mutations associated with the FCHL phenotype and those with increased LDL-C levels. Minocci et al. reported that up to $5 \%$ of cases with FCHL and predicted dysfunctional $L D L R$ had to be reclassified as familial hypercholesterolemia with elevated TG levels, whereby additional genetic variants and environmental factors were responsible for the elevated TG concentrations $^{11,22}$. An additional example of these interactions is the loss-of-function in lipoprotein lipase (LPL), APOA5 and GCKR, which have known to contribute to elevated TG levels, and which interact with additional genetic variants in other genes that increase LDL-C concentrations in $\mathrm{FCHL}^{22}$. Genome-wide scans have also demonstrated a strong link between the angiopoietin-like protein 3 gene (ANGPTL3) and plasma TG levels in $\mathrm{FCHL}^{23}$. ANGPTL3 is a secretory protein that affects plasma TG levels by reversibly inhibiting the catalytic activity of LPL; studies in both animal and human models have shown that inactivation of ANGPTL3 leads to a decrease in TG, high-density lipoprotein-cholesterol (HDL-C), and LDL-C levels, which might diminish the risk of atherosclerotic cardiovascular disease $\mathrm{e}^{14}$. However, the role of alterations in
ANGPTL3 in FCHL patients and its potential therapeutic role have not been determined ${ }^{24}$.

Additional SNPs have been identified for specific populations in relation to $\mathrm{FCHL}$ and metabolic comorbidities. Huertas-Vázquez et al. demonstrated that the rs7903146 and rs 12255372 variants in TCF7L2 are associated with TG concentrations and T2D in Mexicans with FCHL as well as the 20q12-q131 locus, which is explained by HNF $4 \alpha$ variants in Mexican and Finnish subjects with $\mathrm{FCHL}^{25}$. Two novel associations have been recently described for apoB levels at rs1424032 in 16q21, a highly conserved non-codifying region, and rs1349411 12p13.31, which included the $A P O B E C 1$ gene, implicated in the edition of apoB mRNA in the small intestine ${ }^{1}$. Alterations in several metabolic pathways have been identified as potential candidates to further describe metabolic alterations in FCHL. Altered pathways in $\mathrm{FCHL}$ have been reported in the APOA1-C3-A4-A5 gene cluster, which has been linked to HDL-c and TG levels, as well as $L P L$, LCAT, and TNFRSF1B ${ }^{10,11,22}$. SNPs in some of these loci have been linked to both TG and cholesterol fluctuations, with recent reports also suggesting a role for a highly disruptive p.Tyr125Cys SNP in SLC25A40, which encodes a mitochondrial solute transporter evaluated in Seattle kindred ${ }^{26}$.

\section{PATHOPHYSIOLOGY OF FCHL}

FCHL comprises both hyperapobetalipoproteinemia and normal or elevated apoB synthesis 27,28 . Alterations in both secretion and degradation of apoB particles have been encountered in $\mathrm{FCHL}$ patients and have been linked to IR, decreased apoB clearance rate, and increased expression of molecules that downregulate the LDL receptor ${ }^{2,12,30}$. Imbalance between de novo lipogenesis and $\beta$-oxidation is a hallmark of $\mathrm{FCHL}$, resulting in hepatic fat accumulation and very low-density lipoprotein (VLDL) overproduction $^{31}$. Adipose tissue dysfunction has been linked with an increase in free-fatty acid (FFA) levels and efflux of FFA toward the liver, leading to an increased rate of lipoprotein synthesis ${ }^{2,3}$. It is known that increased levels and production of apoC-II and C-III are determinants of kinetics and plasma concentrations of TG-rich lipoproteins (TRLs), including VLDL1 and $2^{32}$. The APOCIII gene has also been linked to states of IR and T2D, both of which are frequent in $\mathrm{FCHL}$ 
patients. FCHL has also been characterized by lower intestinal cholesterol absorption and higher cholesterol synthesis independent of body mass index (BMI) in comparison to primary hypercholesterolemia of genetic origin ${ }^{33}$. Unfavorable lipid profiles and increased postprandial lipemia have been linked to higher cardiovascular risk in $\mathrm{FCHL}^{34}$. A study by Almeda-Valdes et al. determined that the incremental area under the curve of postprandial lipemia in $\mathrm{FCHL}$ patients is determined by fasting apoB-48 levels 35,36 and potentiated by the presence of abdominal obesity. This study also proposed that apoA-V was associated with VLDL and chylomicron production in FCHL subjects.

The role of USF1 in the pathogenesis of FCHL has not been completely explained. Inactivation of USF 1 in mice leads to protection for diet-induced dyslipidemia, obesity, NAFLD, and atherosclerosis; the proposed mechanism has been linked to increased TG uptake by brown adipose tissue through an LPLdependent mechanism, which increases adrenergic response and thermogenesis ${ }^{16}$. USF1 knockout mice (USF1 ${ }^{-1-}$ ) preserved a normal lipid profile when exposed to a high-fat and -carbohydrate diet; in addition, this group showed an enhanced insulin sensitivity and reduced liver steatosis compared with $\mathrm{USF}^{+/+}$type mice ${ }^{16}$. The findings of USF-1 downregulation in animal models are similar to those observed in humans in whom improved insulin sensitivity, atheroprotective lipid profiles, and decreased atherosclerosis were associated with reduced USF1 mRNA expression ${ }^{16}$. Plaisier et al. compared USF1 expression patterns in subcutaneous adipose tissue from $\mathrm{FCHL}$ patients compared to healthy controls, demonstrating higher USF1 expression in affected subjects ${ }^{1}$. Wu et al. developed two overexpression USF1 models in mice; both liver and systemic USF1 overexpression models showed adverse metabolic phenotypes including obesity, worsened lipid profile, and higher glucose/insulin ratio $^{37}$. These observations suggest a role for USF1 in the pathophysiology of $\mathrm{FCHL}$; however, identification of precise mechanisms requires functional studies on human subjects with FCHL with and without USF1 variants that can be later confirmed in controlled studies in animal models.

In FCHL, there is an upregulation of thioredoxins, which are disulfide reductases responsible for regulating redox reactions, and confers anincreased oxidative stress with reduced glutathione levels associated to IR. Both the increase in oxidative stress damage and IR contribute to atherosclerosis and potentially to increased cardiovascular risk in FCHL patients $^{38}$. Most FCHL patients have increased sdLDL and apoB levels for all levels of IR in comparison to controls, adjusted by HOMA-IR and BMI'; this supports the concept that the etiology of the lipid phenotype in FCHL is a result of additive effects of genetic determinants with modulation by BMI and IR. Cardiovascular risk has also been associated to IR in FCHL. Subjects with FCHL have been reported to have increased vascular inflammation and metabolic activity in spleen, bone marrow, and liver as measured by ${ }^{18} \mathrm{~F}$-fluorodeoxyglucose positron-emission tomography/computed tomography imaging ${ }^{39}$. In addition, Carratala et al. showed that subjects with FCHL had increased plasminogen activator inhibitor type 1 (PAI-1) levels, which correlated with IR, MS components and increased carotid intima thickness, all of which are markers of increased cardiovascular risk $^{17,39-42}$.

Along with TRL, diminished lipoprotein clearance associated to IR-mediated decreased LPL activity, leads to sdLDL and intermediate-density lipoprotein particle accumulation, both of which are highly atherogenic and easily oxidized, contributing to its entry into subendothelial pathways ${ }^{3,43}$. Decreased adiponectin levels in the setting of IR have been linked to higher levels of apoB and VLDL particles, further contributing to atherogenesis ${ }^{44}$, which might be feasible in the setting of FCHL. Fibroblast growth factor 21 (FGF-21) is also implicated in the metabolism and kinetics of TRLs, causing an increase in insulin-induced CD36 and LPL-mediated catabolism of TRLs in white and brown adipose tissue and a reduction of serum TG concentrations. It could be hypothesized that the FGF-21 physiologic activity may be decreased in $\mathrm{FCHL}$, but this has not been shown in animal or human subjects ${ }^{45}$. There is also evidence that PCSK9 concentrations are elevated in $\mathrm{FCHL}$ and contribute to the impaired catabolism of apoB ${ }^{12}$. PSCK9 induces degradation and downregulation of the LDL receptor through resistin and other pro-inflammatory cytokines ${ }^{2,12}$ and is as well one of the factors contributing to the hyperapolipoproteinemia in $\mathrm{FCHL}$ and a possible target for therapies as discussed later. 


\section{COMORBIDITIES AND CARDIOVASCULAR RISK IN FCHL}

\section{Metabolic comorbidities in FCHL}

FCHL has been associated to numerous metabolic diseases and comprises metabolic and biochemical abnormalities not unlike T2D, NAFLD, and the MS. FCHL has also been linked to an increased cardiovascular risk, particularly with $\mathrm{CAD}^{3}$. MS shares several pathophysiological alterations with $\mathrm{FCHL}$, including elevated TG levels, impaired glucose tolerance, increased cardiovascular risk, and the comorbid presence of obesity and hypertension. However, in contrast to MS, FCHL subjects consistently show apoB levels $>90^{\text {th }}$ percentile, while in patients with MS apoB may be high, normal, or even decreased. FCHL onset occurs earlier and hereditary traits are more evident, while for MS lifestyle plays a more prominent role than genetics ${ }^{3}$. In a recent study, Skoumas et al. examined the relationship between FCHL and MS, demonstrating that apoB levels were higher for $\mathrm{FCHL}$ patients, despite many similar features in both ${ }^{11}$.

FCHL has been shown to carry an increased risk of incident T2D, conferring a higher metabolic and cardiovascular burden for patients with the disease. However, FCHL studies that evaluate cardiovascular risk often omit population with comorbid T2D, making excess risk estimations unfeasible $e^{1,40}$. The shared genetic background in FCHL has also been suggested by the evidence of association of $\mathrm{FCHL}$ with variants in HNF4 $\alpha$ and TCF7L $2^{1,25,40,46}$. FCHL has been shown to share common pathophysiological mechanisms with T2D including muscle and adipose tissue IR, as well as impaired insulin-mediated suppression of hepatic VLDL production ${ }^{40}$.

An increased risk of hepatic steatosis has been observed in $\mathrm{FCHL}$, with consistent associations for both NAFLD and non-alcoholic steatohepatitis (NASH) and up to $20-37 \%$ of the variability in intrahepatic fat content attributable to genetic factors in $\mathrm{FCHL}^{40,47,48}$. Increased hepatic visceral fat explains the change in serum TG levels in relation to changes in alanine aminotransferase levels for FCHL patients ${ }^{48}$. Brouwers et al. described that fatty liver occurrence was significantly higher for $\mathrm{FCHL}$ patients and their normolipidemic family members when compared to their spouses, who were used as control subjects, and determined that subcutaneous and intravisceral fat were predictors of intrahepatic fat content $^{2}$. Recent studies suggest that genetic polymorphisms in USF1 (rs6427573 and rs2516839), which have been linked to $\mathrm{FCHL}$, have an increased independent risk of NAFLD when compared to controls in Chinese population ${ }^{49}$. Due to the role of USF1 in the transcriptional regulation of hepatic lipogenesis, mice with USF1 overexpression could be used to understand the role of USF1 in the setting of hepatosteatosis and IR and epigenetic studies could contribute to the understanding of the role of posttranslational modifications in the setting of hepatosteatosis in $\mathrm{FCHL}$ patients ${ }^{17}$. Identification of additional mutations that explain intrahepatic fat accumulation, NAFLD, and NASH in FCHL patients remains largely unexplored and requires further evaluation and validation in other ethnic groups.

\section{Cardiovascular risk in FCHL}

$\mathrm{FCHL}$ is strongly associated with premature CAD, with up to $10-14 \%$ of patients with premature CAD having comorbid $\mathrm{FCHL}^{22}$. A patient diagnosed with $\mathrm{FCHL}$ has 1.7-10-fold higher risk of CAD compared to the average population 20 years after the initial diagnosis ${ }^{22,50}$. Wiesbaue et al. demonstrated that $38 \%$ of premature $\mathrm{MI}$ survivors had $\mathrm{FCHL}$, and a similar study including 706 participants with FCHL reported a CAD prevalence of $15.3 \%$, describing that disease presentation was independent of age, sex, or presence of $\mathrm{T}^{2} \mathrm{D}^{6}$. Cardiovascular risk in patients with hypertriglyceridemia is also increased, especially in the setting of older age, tobacco use, and hypertension and decreased HDL-C levels ${ }^{11}$. Among FCHL patients, males are more susceptible to inherit and develop the lipid disorder independent of lipid profile, which might also account for the increased risk ${ }^{24}$. Elevated expression of CD11b, a marker of fasting and postprandial leucocyte activation, has been previously reported for FCHL subjects and has been associated to increased cardiovascular risk in subjects with FCHL and comorbid T2D ${ }^{51-55}$.

\section{THERAPEUTIC APPROACH IN FCHL}

As of the writing of this review, no specific clinical trials, guidelines, or algorithms have been developed for the management of FCHL. However, some 
guidelines such as the 2016 ESC/EAS Guidelines for the Management of Dyslipidemias suggest that it would be managed as a particular primary lipid disorder and an atherogenic dyslipidemia ${ }^{56,57}$. However, the recommendation is lacking since it considers the oligogenic nature of the disease but does not offer specific comments regarding the particular cardiovascular risk in $\mathrm{FCHL}$ and how it might interact with metabolic comorbidities which also increase CV risk; furthermore, the guideline does not offer precise recommendations regarding family studies and early initiation of treatment in susceptible individuals.

A reasonable initial step in the management of a patient with $\mathrm{FCHL}$ includes controlled interventions targeting modifiable cardiovascular risk factors including smoking, alcoholism, overweight, and obesity ${ }^{12}$. Mateo-Gallego et al. showed that a weight loss of $5 \%$ of total weight in overweight adults with FCHL significantly reduces TG and non-HDL cholesterol levels at 3 and 6 months ${ }^{58}$. This justifies the role of weight loss in overweight patients with $\mathrm{FCHL}$ to complement lipid-lowering therapy in $\mathrm{FCHL}^{59}$. Evidence and recommendations regarding management of specific risk factors in FCHL are insufficient and call for the development of intervention-based evaluations aiming at describing the role and magnitude of these treatments and their impact on lipid profile and metabolic burden.

The decision of using either a statin, a fibrate, or a combination of both as the first-line therapy in $\mathrm{FCHL}$ is highly dependent on the predominant lipid alteration at diagnosis. However, it has been shown that the use of statins in comparison to fibrates as the firstline therapy significantly improves the lipid profile and increases the likelihood of reaching lipid targets in patients with $\mathrm{FCHL}^{60,61}$. Furthermore, statins have been shown to decrease significantly the levels of total cholesterol, LDL-C, apoB, non-HDL-C, and VLDL particles and remnants in comparison to fibrates, which are more effective at decreasing TG and increasing HDL-C levels in $\mathrm{FCHL}$ patients ${ }^{61}$. The effect of statin therapy on lipoprotein kinetics was evaluated by Le et al., who demonstrated that rosuvastatin significantly decreases LDL-C, apoB-100, and TG levels and increases the fractional catabolic rate of LDL and apoB-100 in a dose-dependent manner and thus cholesterol biosynthesis but does not have effect on apoB-100 production or HDL kinetics. This indicates that high-intensity statins in $\mathrm{FCHL}$ patients are effective at decreasing LDL apoB-100 levels and thoroughly modify lipoprotein profile ${ }^{62}$. As mentioned earlier, the inhibition of apoB production may also have beneficial roles in preventing hepatosteatosis and improving beta-oxidative pathways, which suggests a potential role to investigate the therapeutic effect of VLDL reduction in $\mathrm{FCHL}$ patients ${ }^{42}$.

FCHL confers an increased risk of premature cardiovascular disease partly due to a rise in the accumulation of atherogenic particles, which may require the use of moderate- to high-intensity statin. Rosuvastatin increases the catabolism of sdLDL apoB-100 levels without changes in the conversion of TRL apoB100 to sdLDL, though at a lower rate than large buoyant LDL-C ${ }^{63}$. Additional cardiovascular risk has been associated to increased postprandial lipemia in $\mathrm{FCHL}$ patients; despite its efficacy in SdLDL and EDL-C reduction, statin therapy has not shown modifications on postprandial lipemia for most FCHL patients, except for MTP-493G/T carriers, in whom a greater reduction of postprandial lipemia has been associated with the use of atorvastatin in comparison to noncarriers $^{36}$. A particular concern of statin therapy in FCHL patients is the associated increase in incident T2D risk with statin use. Skoumas et al. showed that the risk of incident T2D did not increase with statin use or statin intensity in $\mathrm{FCHL}$ patients; these findings were confirmed by their group after a 10-year followup in which no significant differences in T2D incidence with statin use were observed between subjects with $\mathrm{FCHL}$ and controls. These observations suggest that the risk/benefit analysis for statin use in FCHL should have considerations similar to the rest of the population in terms of the statin-associated incident T2D risk $^{45,36}$.

Current guidelines suggest that FCHL patients should be managed according to LDL levels to decrease cardiovascular risk as recommended by European guidelines, by which these levels are considered a condition of high CV risk ${ }^{64-66}$ (Table 2). However, patients with $\mathrm{FCHL}$ often present hypertriglyceridemia, which may decrease the reliability of LDL-C estimation by the Friedewald equation; thus, alternative goals focusing on non-HDL cholesterol and apoB levels are required. Sniderman et al. conducted a meta-analysis to investigate whether apoB or non-HDL-C increased the predictive power of $L D L-C$. They reported that during a 
Table 2. 2016/European Society of Cardiology/European Atherosclerosis Society for treatment of lipid disorder recommendations.

\begin{tabular}{|c|c|c|c|}
\hline CV risk & Features & LDL-C target concentration & $\frac{N}{2}$ \\
\hline Low risk & SCORE $<1 \%$ for 10 -year risk of fatal CVD & $\begin{array}{l}<190 \mathrm{mg} / \mathrm{dL} \text { lifestyle intervention, } \\
\text { drug if uncontrolled }\end{array}$ & consider \\
\hline Moderate risk & SCORE is $>1 \%$ and $<5 \%$ for 10 -year risk of fatal CVD & $\begin{array}{l}100-<155 \mathrm{mg} / \mathrm{dL}, \text { Lifestyle interve } \\
\text { consider drug if uncontrolled }\end{array}$ & ention, \\
\hline High risk & $\begin{array}{l}\text { Markedly elevated single risk factors, in particular } \\
\text { cholesterol }>8 \mathrm{mmol} / \mathrm{L}(>310 \mathrm{mg} / \mathrm{dL} \text { ) (e.g., in } \mathrm{FH} \\
\text { and } \mathrm{FCHL} \text { ) or BP }>180 / 110 \mathrm{mmHg} \text {. Most other } \\
\text { people with DM (some young people with type } 1 \\
\text { diabetes may be at low or moderate risk). Moderate } \\
\text { CKD. SCORE } \\
>5 \% \text { and }<10 \% \text { for } 10 \text {-year risk of fatal CVD }\end{array}$ & $\begin{array}{l}70-<100 \mathrm{mg} / \mathrm{dL} \\
\text { Non-HDL-C }<130 \mathrm{mg} / \mathrm{dL}, \text { ApoB }<\end{array}$ & $90 \mathrm{mg} / \mathrm{dL}$ \\
\hline Very high risk & $\begin{array}{l}\text { Documented CVD, clinical or unequivocal on imaging, } \\
\text { previous myocardial infarction, coronary } \\
\text { revascularization, coronary artery bypass graft } \\
\text { surgery, stroke and transient ischemic attack, and } \\
\text { peripheral arterial disease. DM with target organ } \\
\text { damage, severe CKD. SCORE > 10\% for 10-year risk } \\
\text { of fatal CVD }\end{array}$ & $\begin{array}{l}<70 \mathrm{mg} / \mathrm{dL} \\
\text { Non-HDL-C }<100 \mathrm{mg} / \mathrm{dL}\end{array}$ & 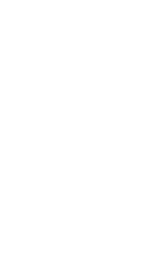 \\
\hline
\end{tabular}

CV: cardiovascular, CVD: cardiovascular disease, HDL-C: high-density lipoprotein cholesterol, T1D: type 1 diabetes, T2D: type 2 diabetes, CKD: chronic kidney disease, SCORE: systematic coronary risk estimation, FH: familial hypercholesterolemia, FCHL: familial combined hyperlipidemia.

10-year period, a strategy focused on controlling non-HDL-C could prevent 300,000 more cardiovascular events than one directed to LDL-C; and a strategy focused on controlling apoB could prevent 500,000 more cardiovascular events than one directed to $\mathrm{LDL}-\mathrm{C}^{67}$. Therefore, targets focused on non$\mathrm{HDL}$ cholesterol $<130 \mathrm{mg} / \mathrm{dL}$ and apoB $<90 \mathrm{mg} / \mathrm{dL}$ should be considered in patients with $\mathrm{FCHL}^{65}$.

Evidence beyond the use of statins for cholesterol management in $\mathrm{FCHL}$ patients has not been extensively evaluated. Ezetimibe can be added to treatment in cases where LDL-C decrease is refractory to statins monotherapy to improve the prognosis in endpoint CAD event as shown in the IMPROVE-IT study ${ }^{68}$. Recent analyses suggest to reconsider the use of ezetimibe and bile acid sequestrants in primary prevention in patients with true statin intolerance and in those patients in whom the goal levels of LDL-C cannot be achieved with maximum statin doses ${ }^{69}$. Evidence from large trials has shown that iPCSk9 is highly effective at reducing $\mathrm{LDL}-\mathrm{C}$ and non-HDL-C levels; they are currently indicated for patients who do not reach lipid target after receiving maximum statin doses and with additional therapy, or patients who have statin intolerance ${ }^{70,71}$. Despite its promising role, the effect of additional LDL-C reduction with iPCSk9 should be evaluated in terms of its effect in cardiovascular risk reduction for $\mathrm{FCHL}$ patients and its impact on specific lipoprotein metabolism and kinetics.

Because $\mathrm{FCHL}$ is also characterized by hypertriglyceridemia, specific measures to control TG concentrations should be taken for adequate management (Table 3). Non-pharmacologic therapy includes glycemic control, avoidance of medication that increases lipid levels, limitation of alcohol intake, avoidance of simple carbohydrates, low-fat diet $(<30 \%$ of total daily caloric intake), and weight loss in patients who are overweight or obese ${ }^{66,72}$. With regard to pharmacological therapy, the efficacy of fibrates, $\Omega-3$ fatty acids, and statins has been demonstrated in clinical trials for the management of $\mathrm{FCHL}$ patients ${ }^{73-75}$. A meta-analysis by Guo et al. demonstrated that the concomitant use of a fibrate and a statin is recommended for the management of $\mathrm{FCHL}$ subjects ${ }^{76}$, and an algorithm proposed by Ellis et al. ${ }^{12}$ suggests that pharmacologic treatment for elevated TG levels in $\mathrm{FCHL}$ patients with levels $>180 \mathrm{mg} / \mathrm{dL}$ should be started after reaching targets of LDL-C and apoB by statin treatment with or without ezetimibe.

Evidence from randomized, controlled clinical trials should be generated in $\mathrm{FCHL}$ population to assess the 
Table 3. Therapeutic goals and treatment strategies in hypertriglyceridemia with FCHL.

\begin{tabular}{|c|c|c|c|}
\hline TG level & Therapeutic goal & Therapeutic strategies & $\frac{N}{L}$ \\
\hline $\begin{array}{l}\text { Borderline high } \\
(150-199 \mathrm{mg} / \mathrm{dL})\end{array}$ & Achieve LDL-C target, and apoB levels & Non-pharmacologic strategies & 离 \\
\hline $\begin{array}{l}\text { High (200-499 } \\
\text { mg/dL) }\end{array}$ & Achieve LDL-C and apoB target, non-HDL-C goal & $\begin{array}{l}\text { Non-pharmacologic strategies } \\
\text { If treatment for LDL-C (statin) } \\
\text { achieve goal, consider: } \\
\text { Fibrate, niacin, } \Omega-3 \text { fatty acids }\end{array}$ & does not \\
\hline $\begin{array}{l}\text { Very high } \\
\quad(>500 \mathrm{mg} / \mathrm{dL})\end{array}$ & $\begin{array}{l}\text { Reduce triglycerides to prevent acute pancreatitis. } \\
\text { Achieve LDL-C target and non-HDL-C. Investigate } \\
\text { secondary causes of elevated TG levels, elevated TG } \\
\text { unlikely due to FCHL }\end{array}$ & $\begin{array}{l}\text { Pharmacologic treatment } \\
\text { Fibrates are preferred or Niacin, } \\
\text { acids, and non-pharmacologic }\end{array}$ & $\Omega-3$ fatty \\
\hline
\end{tabular}

TG: triglycerides, HDL-C: high-density lipoprotein cholesterol, LDL-C: low-density lipoprotein cholesterol, FCHL: familial combined hyperlipidemia, TG: triglycerides

Table 4. Areas of opportunity to improve recognition, standards of care, and research in familial combined hyperlipidemia.

\section{Areas of opportunity in $\mathrm{FCHL}$}

1. Lack of recognition of FCHL and its associated comorbidities by primary care physicians

2. Lack of recognition of associated cardiovascular risk and measures to decrease risk burden

3. Imprecise diagnosis often leads to a lack of family screening, which, in turn, delays treatment in affected individuals

4. Under treatment associated to lack of precise diagnosis or unrecognized possible metabolic and cardiovascular complications

5. Patients are not thoroughly followed, which impairs the ability to influence outcomes and decrease morbidity

6. Studies lack consistent definitions, which makes comparisons across studies difficult

7. Comparative studies of combined treatment strategies are required to improve outcome-oriented treatment algorithms

8. GWAS and EWAS are required to investigate common and rare genetic variants for FCHL in other populations

9. Metabolomics, proteomics, systems biology, and epigenetic studies are required to further the understanding of the pathophysiology of FCHL

10. Follow-up studies are required to evaluate cardiovascular and metabolic risk and assess methods for risk prediction in $\mathrm{FCHL}$

FCHL: familial combined hyperlipidemia

efficacy of new treatments and determine the specific role of statin treatment intensity and fibrate use to improve therapeutic indications. Treatment evaluations should also focus on prevention of metabolic and cardiovascular complications in prospective longterm follow-ups. Given the shared genetic and pathophysiological features between FCHL and T2D, the role of insulin-sensitizing therapy should be evaluated in physiological assessments for human subjects and randomized, controlled clinical trials to determine its utility 40 .

In summary, $\mathrm{FCHL}$ is a common disorder and the most prevalent primary dyslipidemia in the western world. Despite the extensive accumulated knowledge, $\mathrm{FCHL}$ is not usually considered as a first diagnostic choice because of a lack of awareness of its existence among primary care physicians. Therefore, $\mathrm{FCHL}$ is frequently undiagnosed, mostly due to its shifting clinical variability and the heterogeneity of diagnostic criteria, which leads to underreporting of its prevalence in epidemiological studies. This is significant since treatment is often delayed in affected family members that have not been evaluated. Areas of opportunity to improve recognition, standards of care, and research related to genetics, pathophysiology, cardiovascular risk, and management abound and call for further studies to confirm previous findings and increase awareness of this often neglected lipid disorder (Table 4). FCHL is a well-defined oligogenic 
Table 5. Comparison between FCHL and familial hypertriglyceridemia as differential diagnosis of primary lipid disorders with elevated TG levels.

\begin{tabular}{|c|c|c|c|}
\hline Features & $\mathrm{FCHL}$ & Familial hypertriglyceridemia & $\stackrel{4}{2}$ \\
\hline $\begin{array}{l}\text { Former } \\
\text { designations }\end{array}$ & $\begin{array}{l}\text { Familial mixed hyperlipidemia, familial combined } \\
\text { hyperlipoproteinemia, familial combined } \\
\text { hypercholesterolemia-hypertriglyceridemia }\end{array}$ & Type 4 hyperlipidemia & 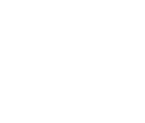 \\
\hline $\begin{array}{l}\text { Main lipoprotein } \\
\text { disturbances }\end{array}$ & Elevated LDL-C and VLDL & Elevated VLDL & \\
\hline Typical onset & Adolescence & Adult age & $\frac{c}{s}$ \\
\hline Clinical features & $\begin{array}{l}\text { Family history of coronary artery disease, } \\
\text { cholesterol and triglyceride levels }>90^{\text {th }} \text { percentile, } \\
\text { ApoB }>90^{\text {th }} \text { percentile, one family member with } \\
\text { TC }>90^{\text {th }} \text { percentile, one family member with TG } \\
>90^{\text {th }} \text { percentile, one family member with } T C \text { and } \\
\text { TG }>90^{\text {th }} \text { percentile }\end{array}$ & $\begin{array}{l}\text { TG levels > } 200 \mathrm{mg} / \mathrm{dL} \text {, Normal or } \\
\text { LDL levels, decreased HDL levels, } \\
\text { decreased apoB levels, } \\
\text { cholesterol:triglyceride ratio } 1: 5 \mathrm{v} \\
\text { reach } 1000 \mathrm{mg} / \mathrm{dL}\end{array}$ & $\begin{array}{l}\text { decreased } \\
\text { Normal or } \\
\text { when TGs } \\
\frac{c}{\frac{c}{n}}\end{array}$ \\
\hline $\begin{array}{l}\text { Association with } \\
\text { CVD }\end{array}$ & +++ & ++ & $\frac{\sqrt{n}}{\frac{\delta}{d}}$ \\
\hline Prevalence & $1 / 40$ & $1 / 20$ & $\frac{\varsigma}{4}$ \\
\hline $\begin{array}{l}\text { Contribution of } \\
\text { secondary } \\
\text { factors }\end{array}$ & Obesity, IR, Metabolic Syndrome, T2D, NASH & $\begin{array}{l}\text { Obesity, T2D, hypertension, pancre } \\
\text { hyperuricemia, IR }\end{array}$ & eatitis, \\
\hline Genetic features & Oligogenic & Variable & $\stackrel{\circ}{2}$ \\
\hline Genetic causes & $\begin{array}{l}\text { TXNIP, RXRA, CRABP2, ATF6, USF1, ANGPLT3, } \\
\text { TCF7L2, APOA5, APOE }\end{array}$ & LPL, APOCII, APOA5, GPIHBP1, LMF & 1 \\
\hline $\begin{array}{l}\text { Current } \\
\text { treatment }\end{array}$ & Statins, ezetimibe, fibrates & Fibrates, niacin, omega-3 fatty acic & ds, fish oil \\
\hline $\begin{array}{l}\text { Future } \\
\text { treatments }\end{array}$ & PCSK9 inhibitors, mipomersen & & $\frac{1}{8}$ \\
\hline
\end{tabular}

HDL: high-density lipoprotein, LDL-C: low-density lipoprotein cholesterol, FCHL: familial combined hyperlipidemia, TG: triglycerides, VLDL: very low-density lipoprotein, ApoB: apolipoprotein B, TC: total cholesterol, T2D: type 2 diabetes, NASH: non-alcoholic steatohepatitis, IR: insulin resistance.

primary lipid disorder with a fluctuating lipid profile, increased cardiovascular risk, and comorbidity with other metabolic conditions such as T2D, NASH, and MS. It is unlikely that a similar phenotype could be found by chance in all patients who have been diagnosed with FCHL, thus, making the case for FCHL as an isolated lipid disorder. FCHL is a critical differential diagnosis in the setting of hypertriglyceridemia or a mixed dyslipidemia, particularly in distinguishing between common hypertriglyceridemia, mixed dyslipidemia, FCHL, and FHTG (Table 5).

\section{REFERENCES}

1. Brouwers MC, van Greevenbroek MM, Stehouwer CD, de Graaf J, Stalenhoef AF. The genetics of familial combined hyperlipidaemia. Nat Rev Endocrinol. 2012;8:352-62.

2. van Greevenbroek MM, Stalenhoef AF, de Graaf J, Brouwers MC. Familial combined hyperlipidemia: from molecular insights to tailored therapy. Curr Opin Lipidol. 2014;25:176-82.
3. Mata P, Alonso R, Ruíz-Garcia A, et al. Familial combined hyperlipidemia: consensus document. Semergen. 2014;40:374-80.

4. Jacobson TA, Maki KC, Orringer CE, et al. National lipid association recommendations for patient-centered management of dyslipidemia: part 2. J Clin Lipidol. 2015;9:S1-122.e1.

5. Escobedo-de la Peña J, de Jesús-Pérez R, Schargrodsky H, Champagne B. Prevalence of dyslipidemias in Mexico city and its relation to other cardiovascular risk factors. Results from the CARMELA study. Gac Med Mex. 2014;150:128-36.

6. Skoumas I, Masoura C, Aznaouridis K, et al. Impact of cardiometabolic risk factors on major cardiovascular events in patients with familial combined hyperlipidemia. Circ J. 2013; 77:163-8.

7. Wierzbicki AS, Graham CA, Young IS, Nicholls DP. Familial combined hyperlipidaemia: Under - Defined and under-diagnosed? Curr Vasc Pharmacol. 2008;6:13-22.

8. Relimpio F, Losada F, Pumar A, et al. Relationships of apolipoprotein $B(100)$ with the metabolic syndrome in Type 2 diabetes mellitus. Diabetes Res Clin Pract. 2002;57:199-207.

9. Sahebkar A, Watts GF. New therapies targeting apoB metabolism for high-risk patients with inherited dyslipidaemias: what can the clinician expect? Cardiovasc Drugs Ther. 2013;27:559-67.

10. Ripatti P, Rämö JT, Söderlund S. The contribution of GWAS loci in familial dyslipidemias. PLoS Genet. 2016;12:e1006078.

11. Brahm AJ, Hegele RA. Combined hyperlipidemia: familial but not (usually) monogenic. Curr opin Lipidol. 2016;27:131-40.

12. Ellis KL, Hooper AJ, Burnett JR, Watts GF. Progress in the care of common inherited atherogenic disorders of apolipoprotein $B$ metabolism. Nat Rev Endocrinol. 2016;12:467-84.

13. Aguilar-Salinas CA, Tusie-Luna T, Pajukanta P. Genetic and environmental determinants of the susceptibility of Amerindian 
derived populations for having hypertriglyceridemia. Metabolism. 2014;63:887-94.

14. Sentinelli F, Minicocci I, Montali A, et al. Association of RXRgamma gene variants with familial combined hyperlipidemia: genotype and haplotype analysis. J Lipids. 2013;2013:517943.

15. Kurokawa R. Initiation of Transcription Generates Divergence of Long Noncoding RNA, Long Noncoding RNAs. Switzerland: Springer; 2015. p. 69-91.

16. Laurila PP, Soronen J, Kooijman S, et al. USF1 deficiency activates brown adipose tissue and improves cardiometabolic health. Sci Transl Med. 2016:8:323ra13.

17. Wang Y, Viscarra J, Kim SJ, Sul HS. Transcriptional regulation of hepatic lipogenesis. Nat Rev Mol Cell Biol. 2015;16:678-89.

18. Guo T, Mao Y, Li H, et al. Characterization of the gene expression profile of heterozygous liver-specific glucokinase knockout mice at a young age. Biomed Pharmacother. 2012;66:587-96.

19. Di Taranto MD, Staiano A, D’Agostino MN, et al. Association of USF1 and APOA5 polymorphisms with familial combined hyperlipidemia in an Italian population. Mol Cell Probes. 2015;29:19-24.

20. Auer S, Hahne P, Soyal SM, et al. Potential role of upstream stimulatory factor 1 gene variant in familial combined hyperlipidemia and related disorders. Arterioscler Thromb Vasc Biol. 2012;32:1535-44

21. Berglund L, Brunzell JD, Goldberg AC, et al. Evaluation and treatment of hypertriglyceridemia: an endocrine society clinical practice guideline. J Clin Endocrinol Metab. 2012;97:2969-89.

22. Minicocci I, Prisco C, Montali A, et al. Contribution of mutations in low density lipoprotein receptor (LDLR) and lipoprotein lipase $(\mathrm{LPL})$ genes to familial combined hyperlipidemia $(\mathrm{FCHL})$ : a reappraisal by using a resequencing approach. Atherosclerosis. 2015;242:618-24

23. Tikka A, Jauhiainen $M$. The role of ANGPTL3 in controlling lipoprotein metabolism. Endocrine. 2016;52:187-93.

24. De Castro-Orós I, Cenarro A, Tejedor MT, et al. Common genetic variants contribute to primary hypertriglyceridemia without differences between familial combined hyperlipidemia and isolated hypertriglyceridemia. Circ Cardiovasc Genet. 2014;7:814-21.

25. Huertas-Vazquez A, Plaisier C, Weissglas-Volkov D, et al. TCF7L2 is associated with high serum triacylglycerol and differentially expressed in adipose tissue in families with familial combined hyperlipidaemia. Diabetologia. 2008;51:62-9.

26. Rosenthal EA, Ranchalis J, Crosslin DR, et al. Joint linkage and association analysis with exome sequence data implicates SLC25A40 in hypertriglyceridemia. Am J Hum Genet. 2013; 93:1035-45.

27. Lapierre L, McLeod R. Regulation of hepatic production of lipoproteins containing apolipoprotein B by ER-associated degradation. Future Lipidol. 2007;2:173-84.

28. Morita SY. Metabolism and modification of apolipoprotein Bcontaining lipoproteins involved in dyslipidemia and atherosclerosis. Biol Pharm Bull. 2016;39:1-24.

29. Dewey FE, Gusarova V, Dunbar RL, et al. Genetic and pharmacologic inactivation of ANGPTL3 and cardiovascular disease. N Engl J Med. 2017;377:211-21.

30. Johansen RF, Søndergaard E, Sørensen LP, et al. Basal and insulin-regulated VLDL1 and VLDL2 kinetics in men with Type 2 diabetes. Diabetologia. 2016;59:833-43

31. Lewis GF, Xiao C, Hegele RA. Hypertriglyceridemia in the genomic era: a new paradigm. Endocr Rev. 2015;36:131-47.

32. Ooi EM, Chan DC, Hodson L, et al. TG-rich lipoprotein metabolism in women: roles of apoC-II and apoC-III. Eur J Clin Invest. 2016;46:730-6.

33. Baila-Rueda L, Cenarro A, Lamiquiz-Moneo I, et al. Cholesterol over synthesis markers define familial combined hyperlipidemia versus other genetic hypercholesterolemias independently of body weight. J Nutr Biochem. 2017;53:48-57.

34. Cruz-Bautista I, Mehta R, Cabiedes J, et al. Determinants of VLDL composition and apoB-containing particles in familial combined hyperlipidemia. Clin Chim Acta. 2015;438:160-5.

35. Almeda-Valdes P, Cuevas-Ramos D, Mehta R, et al. Factors associated with postprandial lipemia and apolipoprotein A-V levels in individuals with familial combined hyperlipidemia. BMC Endocr Disord. 2014;14:90.

36. Klop B, Verseyden C, Ribalta J, et al. MTP gene polymorphisms and postprandial lipemia in familial combined hyperlipidemia: effects of treatment with atorvastatin. Clin Investig Arterioscler. 2014:26:49-57

37. Roman TS, Marvelle AF, Fogarty MP, et al. Multiple hepatic regulatory variants at the GALNT2 GWAS locus associated with high-density lipoprotein cholesterol. Am J Hum Genet. 2015; 97:801-15.
38. Martinez-Hervas S, Artero A, Martinez-Ibañez J, et al. Increased thioredoxin levels are related to insulin resistance in familial combined hyperlipidaemia. Eur J Clin Invest. 2016:46:636-42.

39. Toutouzas K, Skoumas J, Koutagiar I, et al. Vascular inflammation and metabolic activity in hematopoietic organs and liver in familial combined hyperlipidemia and heterozygous familial hypercholesterolemia. J Clin Lipidol. 2018;12:33-43.

40. Brouwers MC, de Graaf J, van Greevenbroek MM, et al. Novel drugs in familial combined hyperlipidemia: lessons from Type 2 diabetes mellitus. Curr Opin Lipidol. 2010;21:530-8.

41. Jiang ZG, de Boer IH, Mackey RH, et al. Associations of insulin resistance, inflammation and liver synthetic function with very low-density lipoprotein: the cardiovascular health study. Metabolism. 2016:65:92-9.

42. Conlon DM, Thomas T, Fedotova T, et al. Inhibition of apolipoprotein B synthesis stimulates endoplasmic reticulum autophagy that prevents steatosis. J Clin Invest. 2016;126:3852-67.

43. Castro Cabezas M. Postprandial lipaemia in familial combined hyperlipidaemia. Biochem Soc Trans. 2003;31:1090-3.

44. Dallinga-Thie GM, Kroon J, Borén J, Chapman MJ. TG-rich lipoproteins and remnants: targets for therapy? Curr Cardiol Rep. 2016;18:67.

45. Schlein C, Talukdar S, Heine M, et al. FGF21 lowers plasma triglycerides by accelerating lipoprotein catabolism in white and brown adipose tissues. Cell Metab. 2016;23:441-53.

46. Zeggini E1, Damcott CM, Hanson RL, et al. Variation within the gene encoding the upstream stimulatory factor 1 does not influence susceptibility to Type 2 diabetes in samples from populations with replicated evidence of linkage to chromosome 1q Diabetes. 2006;55:2541-8.

47. Brouwers MC, Bilderbeek-Beckers MA, Georgieva AM, et al Fatty liver is an integral feature of familial combined hyperlipidaemia: relationship with fat distribution and plasma lipids. Clin Sci (Lond). 2007;112:123-30.

48. López-Velázquez JA, Silva-Vidal KV, Ponciano-Rodríguez G, et al The prevalence of nonalcoholic fatty liver disease in the Americas. Ann Hepatol. 2014;13:166-78.

49. Wang Y, Wang BF, Tong J, Chang B, Wang BY. USF-1 genetic polymorphisms confer a high risk of nonalcoholic fatty liver disease in Chinese population. Int J Clin Exp Med. 2015;8: 2545-53.

50. Mehta R, Reyes-Rodríguez E, Yaxmehen Bello-Chavolla O, et al Performance of LDL-C calculated with martin's formula compared to the Friedewald equation in familial combined hyperlipidemia. Atherosclerosis. 2018; on line.

51. de Vries MA, Alipour A, Klop B, et al. Glucose-dependent leukocyte activation in patients with Type 2 diabetes mellitus, familial combined hyperlipidemia and healthy controls. Metabolism. 2015;64:213-7.

52. Alipour A, Valdivielso P, Elte JW, et al. Exploring the value of apoB48 as a marker for atherosclerosis in clinical practice. Eur J Clin Invest. 2012;42:702-8.

53. Fan YM, Hernesniemi J, Oksala N, et al. Upstream transcription factor 1 (USF1) allelic variants regulate lipoprotein metabolism in women and USF1 expression in atherosclerotic plaque. Sci Rep. 2014:4:4650.

54. Amor AJ, Ortega E, Perea $\mathrm{V}$, et al. Relationship between total serum bilirubin levels and carotid and femoral atherosclerosis in familial dyslipidemia. Arterioscler Thromb Vasc Biol. 2017;37: 2356-63.

55. Averna M, Stroes E, Lipid Alterations Beyond LDL Expert Working Group. How to assess and manage cardiovascular risk associated with lipid alterations beyond LDL. Atheroscler Suppl. 2017; $26: 16-24$

56. Nordestgaard BG, Chapman MJ, Humphries SE, et al. Familial hypercholesterolaemia is underdiagnosed and undertreated in the general population: guidance for clinicians to prevent coronary heart disease: consensus statement of the European atherosclerosis society. Eur Heart J. 2013;34:3478-90a.

57. Catapano AL, Graham I, De Backer G et al. 2016 ESC/EAS guidelines for the management of dyslipidaemias. Eur Heart J. 2016;37:2999-3058.

58. Kei AA, Filippatos TD, Tsimihodimos V, Elisaf MS. A review of the role of apolipoprotein C-II in lipoprotein metabolism and cardiovascular disease. Metabolism. 2012;61:906-21.

59. Mateo-Gallego R, Perez-Calahorra S, Cofán M, et al. Serum lipid responses to weight loss differ between overweight adults with familial hypercholesterolemia and those with familial combined hyperlipidemia. J Nutr. 2014;144:1219-26.

60. Martin SS, Abd TT, Jones SR, Michos ED, Blumenthal RS, Blaha MJ. 2013 ACC/AHA cholesterol treatment guideline: what was 
done well and what could be done better. J Am Coll Cardiol. 2014;63:2674-8

61. Arca M, Montali A, Pigna G, et al. Comparison of atorvastatin versus fenofibrate in reaching lipid targets and influencing biomarkers of endothelial damage in patients with familial combined hyperlipidemia. Metabolism. 2007;56:1534-41.

62. Le NA, Diffenderfer MR, Thongtang $N$, et al. Rosuvastatin enhances the catabolism of LDL apoB-100 in subjects with combined hyperlipidemia in a dose dependent manner. Lipids. 2015; 50:447-58

63. Thongtang N, Diffenderfer MR, Ooi EM, et al. Metabolism and proteomics of large and small dense LDL in combined hyperlipidemia: effects of rosuvastatin. J Lipid Res. 2017;58:1315-24.

64. Emerging Risk Factors Collaboration, Di Angelantonio E, Gao P, et al. Lipid-related markers and cardiovascular disease prediction. JAMA. 2012;307:2499-506.

65. Stone NJ, Robinson JG, Lichtenstein AH, et al. 2013 ACC/AHA guideline on the treatment of blood cholesterol to reduce atherosclerotic cardiovascular risk in adults: a report of the American college of cardiology/American heart association task force on practice guidelines. J Am Coll Cardiol. 2014;63: 2889-934.

66. Anderson TJ, Grégoire J, Hegele RA, et al. 2012 update of the Canadian cardiovascular society guidelines for the diagnosis and treatment of dyslipidemia for the prevention of cardiovascular disease in the adult. Can J Cardiol. 2013;29:151-67.

67. Sniderman AD, Williams K, Contois JH, et al. A meta-analysis of low-density lipoprotein cholesterol, non-high-density lipoprotein cholesterol, and apolipoprotein B as markers of cardiovascular risk. Circ Cardiovasc Qual Outcomes. 2011;4:337-45.
68. Cannon CP, Blazing MA, Giugliano RP, et al. Protocol-ezetimibe added to statin therapy after acute coronary syndromes. N Engl J Med. 2015;372:2387-97.

69. Banach M, Nikolic D, Rizzo M, Toth PP. IMPROVE-IT: what have we learned? Curr Opin Cardiol. 2016;31:426-33.

70. AlHajri L, AlHadhrami A, AlMheiri S, AlMutawa Y, AlHashimi Z. The efficacy of evolocumab in the management of hyperlipidemia: a systematic review. Ther Adv Cardiovasc Dis. 2017;11:155-69.

71. Adhyaru BB, Jacobson TA. Role of non-statins, LDL-C thresholds, and special population considerations: a look at the updated 2016 ACC consensus committee recommendations. Curr Atheroscler Rep. 2017;19:29.

72. Estruch R, Ros E, Salas-Salvadó J, et al. Primary prevention of cardiovascular disease with a Mediterranean diet. N Engl J Med. 2013;368:1279-90

73. Akalin Çiftçi G, Ertorun İ, Akalin A, Alataş İÖ, Musmul A. The effects of atorvastatin on antioxidant/antiinflammatory properties of HDLs in hypercholesterolemics. Turk ] Med Sci. 2015:45:345-51.

74. Brandt EJ, Davidson $\mathrm{MH}$. The role of omega-3 fatty acids in dyslipidemias. Combination Therapy in Dyslipidemias. New York, USA: Springer; 2015. p. 45-64.

75. Perk J, de Backer G, Gohlke H, et al. European Guidelines on cardiovascular disease pre-vention in clinical practice (version 2012). The fifth joint task force of the European society of cardiology and other societies on cardiovascular disease prevention in clinical practice (constituted by representatives of nine societies and by invited experts). Atherosclerosis. 2012;223:1-68.

76. Guo J, Meng F, Ma N, et al. Meta-analysis of safety of the coad ministration of statin with fen fibrate in patients with combined hyperlipidemia. Am J Cardiol. 2012;110:1296-301. 